\title{
A Comparative Study on Adipose Tissue of Different Fat-Tailed Sheep
}

\author{
Ren Rui ${ }^{1}$, Cao Xin ${ }^{2}$, A Yimuguli ${ }^{1}$, Dai Hongwei ${ }^{1}$, Xu Hongwei ${ }^{2,}$, Zang Rongxin $^{1, ~ *}$ \\ ${ }^{1}$ College of Life Science and Engineering, Northwest Minzu University, Lanzhou, China \\ ${ }^{2}$ Experimental Center, Northwest Minzu University, Lanzhou, China
}

\section{Email address:}

1016902572@qq.com (Ren Rui),16393213@qq.com(Cao Xin), ayimgu180@qq.com(A Yimuguli),809894874@qq.com (Dai Hongwei), hongweixu2009@qq.com(Xu Hongwei),rxzang2000@163.com(Zang Rongxin)

${ }^{*}$ Corresponding author

\section{To cite this article:}

Ren Rui, Cao Xin, A Yimuguli, Dai Hongwei, Xu Hongwei, Zang Rongxin. A Comparative Study on Adipose Tissue of Different Fat-Tailed Sheep. Science Discovery. Vol. 6, No. 3, 2018, pp. 218-223. doi: 10.11648/j.sd.20180603.23

Received: March 26, 2018; Accepted: June 26, 2018; Published: June 27, 2018

\begin{abstract}
The aim is to understand the structure and composition characteristics of adipose tissue in different fat-tailed sheep, to lay a foundation for the study on the induced deposition of adipose tissue. The number of 6 for each eight-month-old Lanzhou big-tailed sheep, small-tailed Han sheep and Tibetan sheep wether have been selected for the slaughter experiment. Then, the subcutaneous, greater omental, perirenal and caudal fats have been collected for cell morphology studies, while the fat of Lanzhou big-tailed sheep and Tibetan sheep have selected to study composition and content of fatty acid. By comparison: for fat-tailed sheep, the fat is enriched in tail, subcutaneous tissue, and omentum, whereas for less fat-tailed sheep, the fat is enriched in viscera and subcutaneous tissue. The subcutaneous fat of fat-tailed sheep had early maturation, high degree of differentiation, and long growth phase of fat tail. For the less fat-tailed sheep, the subcutaneous fat and perirenal fat type have similar characteristics of differentiation and development, while the tail fat has early maturation. For Lanzhou big-tailed sheep and Tibetan sheep, their major fatty acids have similar characteristics of site distribution.
\end{abstract}

Keywords: Fat Tail Sheep, Adipose Tissue, Morphology, Fatty Acids

\section{不同脂尾型绵羊脂肪组织比较研究}

\author{
任瑞 ${ }^{1}$ ，曹忻 ${ }^{2}$, 阿依木古丽 ${ }^{1}$ ，戴洪伟 ${ }^{1}$ ，徐红伟 ${ }^{2 *}$, 㶓荣釒金 ${ }^{*}$ \\ ${ }^{1}$ 生命科学与工程学院, 西北民族大学, 兰州, 中国 \\ ${ }^{2}$ 实验中心, 西北民族大学, 兰州, 中国
}

\section{邮箱}

1016902572@qq.com（任瑞）, 16393213@qq.com（曹忻）, ayimgu180@qq.com(阿依木古丽）, 809894874@qq.com（戴洪伟）, hongweixu2009@qq.com(徐红伟), rxzang2000@163.com（藏荣金金）

\begin{abstract}
摘要: 旨在了解不同脂尾型绵羊脂肪组织结构和组成特点, 为脂肪组织诱导沉积研究奠定基础。选择 8 月龄兰州大尾羊、 小尾寒羊、藏绵羊羯羊各6只, 进行屠宰试验, 采集皮下、大网膜、肾周、尾部脂肪进行细胞形态研究, 对兰州大尾羊、 藏绵羊脂肪进行脂肪酸组成与含量研究。说明, 脂尾型绵羊以尾部、皮下、大网膜富集脂肪, 而庋脂尾型绵羊以内脏、 皮下富集脂肪; 脂尾型绵羊皮下脂肪发育早，分化程度高，尾脂发育周期长，廋尾型皮下和肾周脂肪有相似分化发育 的特征, 同时尾脂成熟早; 兰州大尾羊和藏绵羊主要脂肪酸具有相似的部位分布特征。
\end{abstract}




\section{1. 引言}

脂肪组织是动物体内重要的储能器官, 哺乳动物脂 肪组织有白色 (或黄色) 脂肪组织和棕色脂肪组织两种 类型。在肉用羊中, 脂肪组织在体内分布及脂肪沉积是 影响胴体品质和肉质风味的关键因素, 而肌内脂肪是影 响大理石纹的物质基础, 也直接参与肉质嫩度、多汁性 和肉品风味的形成 [1], 不同品种绵羊体脂分布有很大差 异, 按照尾脂沉积能力可以将绵羊分为长脂尾型绵羊 (兰 州大尾羊)、短脂尾型绵羊 (小尾寒羊) 、瘦短尾羊 (藏 绵羊) 等[2], 兰州大尾羊尾部脂肪占胴体重 $11.46 \%$ [3], 而藏绵羊沉积以内脏脂肪为主, 脂尾型绵羊在屠宰过程 中尾部脂肪被丢弃, 降低了饲料转化率, 同时一些具有 抗病力强、适应性好的脂尾型品种正在濒临灭绝 [4-5], 通过脂肪定向诱导方式提高肌内脂肪，降低皮下和内脏 脂肪, 改善肉质风味, 成为保护利用尾脂型绵羊的有效 途径之一。为此, 本研究拟采集三种不同脂尾型绵羊脂 肪组织, 从体脂分布、脂肪细胞形态、脂肪酸组成与含 量进行研究, 为脂肪组织定向诱导沉积研究奠定基础。

\section{2. 材料与方法}

\section{1. 试验动物}

甘肃省临夏回族自治州永靖县瑞霖农业科技有限公 司提供舍饲 8 月龄兰州大尾羊、小尾寒羊、藏绵羊羯羊各 6 只。

\section{2. 饲养管理条件和屠宰时间}

表1 试验精料补充饲料配方及营养水平。

\begin{tabular}{lll}
\hline & 指标 & 含量 \\
\hline \multirow{4}{*}{ 原料 } & 玉米 $/ \%$ & 75 \\
& 菜籽渣 $/ \%$ & 5 \\
& 豆粕 $/ \%$ & 15 \\
& 麸皮 $/ \%$ & 5 \\
& 预混料 $/ \%$ & 5 \\
& 合计 & 100 \\
& 粗蛋白质 $/ \%$ & 15.21 \\
& 粗脂肪 $/ \%$ & 2.38 \\
营养水平 & 消化能 $/ \mathrm{MJ} \cdot \mathrm{kg}^{-1}$ & 20.43 \\
& 钲 $/ \%$ & 1.73 \\
& 总磷 $/ \mathrm{g} \cdot \mathrm{d}-1$ & 0.42 \\
\hline
\end{tabular}

注: 每千克预混料中水分 $7.27 \%-8.36 \%$, 铁 $3840 \mathrm{mg}$, 铜 $600 \mathrm{mg}$, 锌 $1500 \mathrm{mg}$, 锰4000mg, 硒50mg, VA150万IU, VD300万IU, VE6000IU。

试验动物分栏舍饲养, 空间宽松, 设有自动饮水处, 圈 外设有活动场所, 定期使用 $0.5 \%$ 强力消毒灵喷酒羊体、羊圈, 使用石灰粉对通道、隔离带进行消毒, 人员、车辆出入实行 强制消毒, 羊只定期进行免疫注射 (口蹄疫、三联四防疫苗)。

饲养条件良好, 精料每天分两次定时饲喂 (6:30和 14:30）, 粗饲料为青贮饲料, 全天自由采食, 参照羔羊
育肥饲养标准7 8月龄的生长发育需要, 经该养殖场大型 颗粒饲料机械配合生产, 精料原料及营养水平见表1

\section{3. 试验方法}

\subsection{1 屠宰测定方法}

正常饲喂结束后, 所有屠宰羊宰前24h停止饲喂, $2 \mathrm{~h}$ 停止饮水, 选择健康状况良好, 无生理机能紊乱和遗传缺 陷的羊, 采用切断颈动脉的方法放血致死, 人工剥皮, 切 除头蹄, 参照赵有璋羊产肉性能的测定与计算方法测量每 只羊的胴体重和不同部位脂肪的重量。

采集腹部皮下、尾部、肾周、大网膜脂肪用锡箔纸包 好放入液氮罐中, 带回实验室 $-80^{\circ} \mathrm{C}$ 保存, 用于脂肪酸测 定研究; 另各部位采集 $2 \mathrm{~cm}^{3}$ 大小样品用 $4 \%$ 多聚甲醛固定, 用于组织学结构研究。

\subsection{2. 脂肪组织切片制作}

脂肪组织经过冲水、脱水、透明、包埋、切片、摊片、 烤片、染色、封片等步骤制作石蜡组织切片, 使用CellSens Entry显微镜图像软件测量脂肪细胞的周长, 选择切片中具 有典型代表性的 5 个区域, 共测量 50 组细胞大小数据。

\subsection{3. 样品脂肪酸组成测定}

粗脂肪测定采用索氏提取法, 脂肪酸测定参照张明等 [6] 人方法, 气相色谱运行条件：毛细管柱 $(100 \mathrm{~m} \times 0.25 \mathrm{~mm} \times 0.2 \mu \mathrm{m}, \mathrm{SPTM}-2560)$, 柱头压 $240 \mathrm{KPa}$, 氮气 (载气) 流速为 $1.5 \mathrm{ml} \cdot \mathrm{min}-1$; 燃气 (氢气) 流速为 $40 \mathrm{~mL} \cdot \mathrm{min}-1$; 空气 (助燃气) 流速为 $400 \mathrm{ml} \cdot \mathrm{min}-1$, 进样口温 度和FID检测器温度均为 $260^{\circ} \mathrm{C}$; 分流比 $100: 1$, 分流流量 为 $150 \mathrm{ml} \cdot \mathrm{min}-1$; 进样量为 $1 \mu 1$ 。用气象色谱对不同脂尾型绵 羊不同部位脂肪组织进行检测, 每个样品做 2 个平行, 取 平均值为该样品的检测结果, 根据脂肪酸甲酯的标准样品 保留时间来鉴定脂肪酸, 并用峰面积归一法来确定各脂肪 酸的相对百分含量。

\subsection{4. 数据处理}

试验结果经EXCEL预处理后采用SPSS 17.0进行统计 分析, 结果以平均数土标准误表示。

\section{3. 结果}

\section{1. 不同脂尾型绵羊体脂分布比较}

不同脂尾型绵羊体脂分布比较如表2所示, 同样饲喂 条件下, 三种羊宰前活重没有显著差异 $(P>0.05)$, 藏绵 羊、兰州大尾羊胴体重显著高于小尾寒羊 $(P<0.01)$; 同 时, 尾部脂肪含量有兰州大尾羊>小尾寒羊 $>$ 藏绵羊 $(P<0.01)$, 藏绵羊尾部脂肪量很少, 几乎不能称重测量, 尾脂占胴体重有兰州大尾羊尾>小尾寒羊 $(P<0.01)$, 肾 周脂肪占胴体重有藏绵羊>兰州大尾羊>小尾寒羊, 且藏绵 羊和小尾寒羊之间差异显著 $(P<0.05)$; 大网膜脂肪占胴 
体重有藏绵羊 $>$ 兰州大尾羊>小尾寒羊, 且小尾寒羊与藏绵 羊之间差异极显著 $(P<0.01)$ 。

表2 不同脂尾型绵羊脂肪组织分布比较 $(\mathrm{kg})$ 。

\begin{tabular}{llll}
\hline & 小尾寒羊 & 藏绵羊 & 兰州大尾羊 \\
\hline 宰前活重 & $29.47 \pm 0.97$ & $33.13 \pm 1.47$ & $35.57 \pm 1.13$ \\
胴体重 & $13.38 \mathrm{Aa} \pm 0.38$ & $17.17 \mathrm{Bb} \pm 0.75$ & $18.02 \mathrm{Bb} \pm 0.27$ \\
尾脂重 & $0.49 \mathrm{Ab} \pm 0.02$ & $0.00 \mathrm{Aa}$ & $1.82 \mathrm{Bc} \pm 0.13$ \\
肾周脂肪重 & $0.05 \mathrm{Aa} \pm 0.01$ & $0.19 \mathrm{Bb} \pm 0.01$ & $0.15 \mathrm{ABb} \pm 0.03$ \\
大网膜脂肪 & $0.19 \mathrm{Aa} \pm 0.02$ & $0.67 \mathrm{Bb} \pm 0.05$ & $0.56 \mathrm{Bb} \pm 0.04$ \\
尾脂重/胴体重 $(\%)$ & $3.71 \mathrm{Bb} \pm 0.25$ & $0.00 \mathrm{Aa}$ & $10.10 \mathrm{Cc} \pm 0.75$ \\
肾周脂肪重/胴体重 $(\%)$ & $0.35 \mathrm{Aa} \pm 0.04$ & $1.13 \mathrm{Ab} \pm 0.06$ & $0.85 \mathrm{Aab} \pm 0.19$ \\
大网膜脂肪重/胴体重 $(\%)$ & $1.42 \mathrm{Aa} \pm 0.19$ & $3.87 \mathrm{Bb} \pm 0.14$ & $3.11 \mathrm{Bb} \pm 0.21$ \\
\hline
\end{tabular}

注: 表达量标注相同小字母表示差异不显著 $(P>0.05)$; 标注不同大写字母表示差异极显著 $(P<0.01)$; 标注不同小写字母表示差异显著 $(P<$ 0.05 )。

\section{2. 不同脂尾型绵羊脂肪组织大小比较}

通过图2对兰州大尾羊、小尾寒羊和藏绵羊脂肪组织 形态分析发现, 兰州大尾羊和小尾寒羊皮下脂肪比肾周脂 肪大 $(P<0.05)$; 藏绵羊有皮下与肾周脂肪之间大小差异不 显著 $(P>0.05)$ ，皮下脂肪比大网膜脂肪大 $(P<0.05)$; 尾脂有兰州大尾羊>小尾寒羊>藏绵羊 $(P<0.05)$ 。
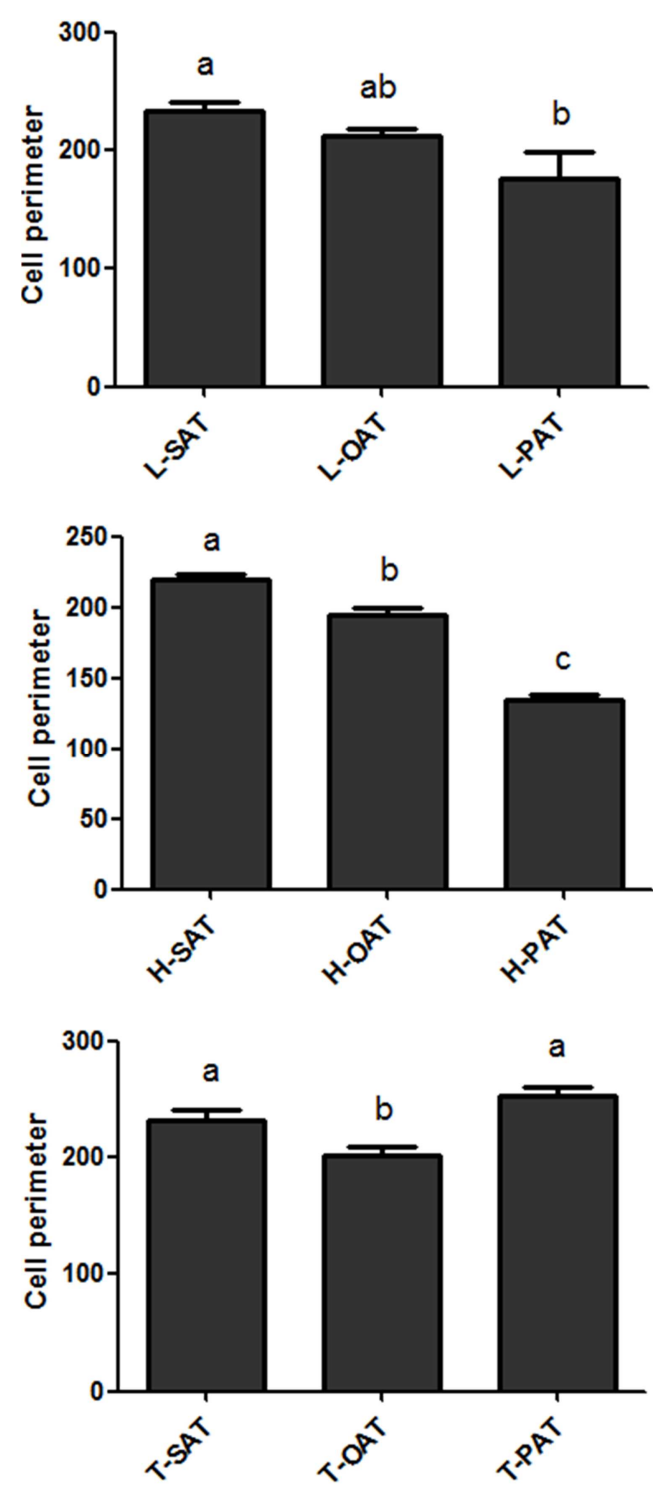

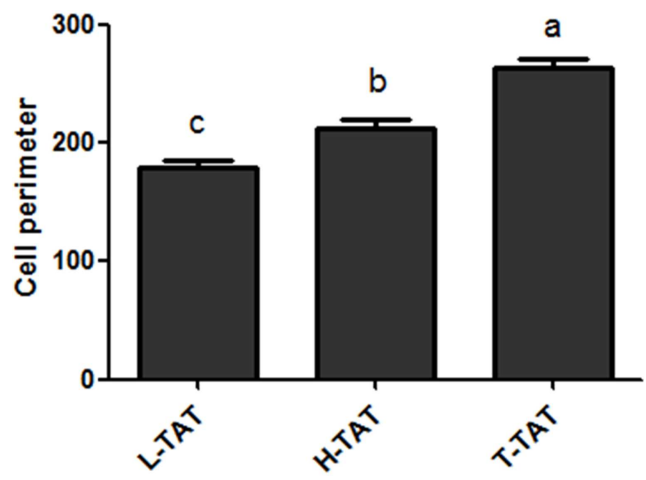

图2 不同脂尾型绵羊脂肪组织大小比较 $(\mu \mathrm{m})$ 。

注: 兰州大尾羊 $(\mathrm{L})$ 、小尾寒羊 $(\mathrm{H})$ 、藏绵羊 $(\mathrm{T})$, 皮下脂肪 $(\mathrm{SAT})$ 、 大网膜脂肪 $(\mathrm{OAT})$ 肾周脂肪 $(\mathrm{PAT})$ 、尾部脂肪 $(\mathrm{TAT})$ 在图中以简写 表示。表达量标注相同小字母表示差异不显著 $(P>0.05)$; 标注不同小 写字母表示差异显著 $(P<0.05)$ 。

\section{3. 不同脂尾型绵羊不同部位脂肪组织组成比较}

\subsection{1. 不同脂尾型绵羊脂肪组织粗脂肪含量比较}

图3可知, 兰州大尾羊、藏绵羊不同解剖部位粗脂肪 含量差异不显著 $(P>0.05)$ 。

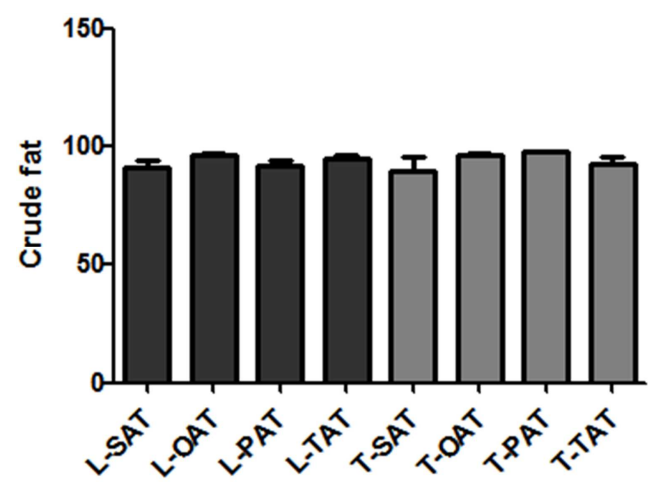

图3 不同脂尾型绵羊脂肪组织粗脂肪含量比较 (\%)。

注: 兰州大尾羊 ( $\mathrm{L}$ ) 、藏绵羊 $(\mathrm{T})$, 皮下脂肪 ( $\mathrm{SAT}$ )、大网膜脂肪 (OAT)肾周脂肪 (PAT)、尾部脂肪（TAT）在图中以简写表示。表达 量标注相同小字母表示差异不显著 $(\mathrm{P}>0.05)$; 标注不同小写字母表示 差异显著 $(\mathrm{P}<0.05)$ 。 
3.3.2. 不同脂尾型绵羊脂肪组织脂肪酸组成与含量比较

表3 不同脂尾型绵羊不同解剖部位脂肪酸含量比较 (\%)。

\begin{tabular}{|c|c|c|c|c|c|c|c|c|}
\hline \multirow{2}{*}{$\begin{array}{l}\text { 脂肪酸名 } \\
\text { 称 }\end{array}$} & \multicolumn{4}{|l|}{ 藏绵羊 } & \multicolumn{4}{|l|}{ 兰州大尾羊 } \\
\hline & 大网膜脂肪 & 皮下脂肪 & 肾周脂肪 & 尾部脂肪 & 大网膜脂肪 & 皮下脂肪 & 肾周脂肪 & 尾部脂肪 \\
\hline C4: 0 & $0.00 \pm 0.00$ & $0.03 \pm 0.03$ & $0.00 \pm 0.00$ & $0.08 \pm 0.08$ & $0.43 \pm 0.35$ & $0.14 \pm 0.14$ & $0.06 \pm 0.04$ & $0.00 \pm 0.00$ \\
\hline C6: 0 & $0.00 \pm 0.00$ & $0.02 \pm 0.01$ & $0.00 \pm 0.00$ & $0.02 \pm 0.02$ & $0.03 \pm 0.02$ & $0.09 \pm 0.05$ & $0.03 \pm 0.02$ & $0.01 \pm 0.00$ \\
\hline C8: 0 & $0.02 \pm 0.00$ & $0.02 \pm 0.01$ & $0.02 \pm 0.00$ & $0.03 \pm 0.01$ & $0.03 \pm 0.00$ & $0.09 \pm 0.06$ & $0.03 \pm 0.02$ & $0.01 \pm 0.00$ \\
\hline C10: 0 & $0.28 \pm 0.03$ & $0.28 \pm 0.06$ & $0.23 \pm 0.03$ & $0.32 \pm 0.06$ & $0.33 \pm 0.07 \mathrm{ab}$ & $0.30 \pm 0.07 \mathrm{ab}$ & $0.21 \pm 0.06 \mathrm{~b}$ & $0.59 \pm 0.25 \mathrm{a}$ \\
\hline C11: 0 & $0.01 \pm 0.00 \mathrm{bc}$ & $0.03 \pm 0.00 \mathrm{~b}$ & $0.01 \pm 0.00 \mathrm{c}$ & $0.04 \pm 0.01 \mathrm{a}$ & $0.03 \pm 0.01 \mathrm{~b}$ & $0.05 \pm 0.02 b$ & $0.04 \pm 0.02 b$ & $0.19 \pm 0.07 \mathrm{a}$ \\
\hline C12: 0 & $0.26 \pm 0.05$ & $0.39 \pm 0.12$ & $0.21 \pm 9.07$ & $0.41 \pm 0.13$ & $0.16 \pm 0.02 b$ & $0.20 \pm 0.05 b$ & $0.15 \pm 0.01 \mathrm{~b}$ & $0.54 \pm 0.22 \mathrm{a}$ \\
\hline C13: 0 & $0.16 \pm 0.01$ & $0.17 \pm 0.02$ & $0.14 \pm 0.02$ & $0.19 \pm 0.02$ & $0.19 \pm 0.03 \mathrm{~b}$ & $0.18 \pm 0.15 b$ & $0.19 \pm 0.02 \mathrm{~b}$ & $0.52 \pm 0.21 \mathrm{a}$ \\
\hline C14: 0 & $4.32 \pm 0.32$ & $4.87 \pm 0.60$ & $4.25 \pm 0.44$ & $4.63 \pm 0.71$ & $2.79 \pm 0.14 \mathrm{~b}$ & $3.53 \pm 0.37 \mathrm{~b}$ & $2.96 \pm 0.9 b$ & $4.25 \pm 0.74 \mathrm{a}$ \\
\hline C15: 0 & $0.70 \pm 0.03 b c$ & $0.81 \pm 0.08 \mathrm{ab}$ & $0.60 \pm 0.03 \mathrm{c}$ & $0.89 \pm 0.06 \mathrm{a}$ & $0.72 \pm 0.11 \mathrm{~b}$ & $0.66 \pm 0.10 \mathrm{~b}$ & $0.66 \pm 0.05 b$ & $1.33 \pm 0.25 \mathrm{a}$ \\
\hline C16: 0 & $24.11 \pm 0.43$ & $25.14 \pm 0.61$ & $24.29 \pm 0.39$ & $23.70 \pm 0.87$ & $19.67 \pm 0.75 b$ & $23.43 \pm 1.45 \mathrm{a}$ & $21.41 \pm 0.76 \mathrm{ab}$ & $20.64 \pm 0.83 \mathrm{ab}$ \\
\hline C17: 0 & $1.78 \pm 0.07$ & $1.72 \pm 0.13$ & $1.62 \pm 0.06$ & $1.98 \pm 0.20$ & $2.07 \pm 0.25$ & $1.49 \pm 0.07$ & $1.64 \pm 0.68$ & $2.14 \pm 0.19$ \\
\hline C18: 0 & $25.24 \pm 0.62 \mathrm{a}$ & $18.30 \pm 0.77 \mathrm{~b}$ & $26.74 \pm 0.81 \mathrm{~b}$ & $13.20 \pm 1.08 \mathrm{c}$ & $24.18 \pm 2.44 \mathrm{ab}$ & $19.94 \pm 1.45 b$ & $28.15 \pm 1.75 \mathrm{a}$ & $11.37 \pm 1.40 \mathrm{c}$ \\
\hline C20: 0 & $0.34 \pm 0.05$ & $0.36 \pm 0.11$ & $0.28 \pm 0.02$ & $0.17 \pm 0.01$ & $0.54 \pm 0.28$ & $0.16 \pm 0.02$ & $0.51 \pm 0.17$ & $0.21 \pm 0.08$ \\
\hline $\mathrm{C} 21: 0$ & $0.71 \pm 0.12$ & $0.80 \pm 0.17$ & $0.61 \pm 0.21$ & $0.71 \pm 0.06$ & $1.13 \pm 0.36$ & $1.26 \pm 0.48$ & $0.71 \pm 0.20$ & $1.07 \pm 0.38$ \\
\hline C22: 0 & $0.20 \pm 0.07$ & $0.20 \pm 0.08$ & $0.25 \pm 0.16$ & $0.28 \pm 0.16$ & $0.68 \pm 0.37$ & $0.24 \pm 0.16$ & $0.16 \pm 0.06$ & $0.18 \pm 0.06$ \\
\hline C23: 0 & $0.00 \pm 0.00$ & $0.12 \pm 0.08$ & $0.03 \pm 0.03$ & $0.03 \pm 0.02$ & $0.30 \pm 0.17$ & $0.01 \pm 0.01$ & $0.02 \pm 0.02$ & $0.14 \pm 0.13$ \\
\hline C24: 0 & $0.00 \pm 0.00$ & $0.11 \pm 0.07$ & $0.03 \pm 0.03$ & $0.07 \pm 0.05$ & $0.11 \pm 0.07$ & $0.02 \pm 0.02$ & $0.07 \pm 0.06$ & $0.01 \pm 0.01$ \\
\hline C14: $\ln 5$ & $0.79 \pm 0.04$ & $0.75 \pm 0.05$ & $0.73 \pm 0.06$ & $0.73 \pm 0.07$ & $0.84 \pm 0.12$ & $0.78 \pm 0.12$ & $0.78 \pm 0.08$ & $1.20 \pm 0.33$ \\
\hline C15: 1 & $0.49 \pm 0.05$ & $0.51 \pm 0.08$ & $0.38 \pm 0.03$ & $0.40 \pm 0.03$ & $0.54 \pm 0.11$ & $0.36 \pm 0.02$ & $0.54 \pm 0.05$ & $0.59 \pm 0.21$ \\
\hline C16: 1 & $3.13 \pm 0.08 b c$ & $3.46 \pm 0.17 \mathrm{ab}$ & $2.82 \pm 0.09 \mathrm{c}$ & $3.77 \pm 0.13 \mathrm{a}$ & $3.45 \pm 0.16 \mathrm{ab}$ & $3.21 \pm 0.08 \mathrm{ab}$ & $2.82 \pm 0.28 \mathrm{~b}$ & $3.78 \pm 2.27 \mathrm{a}$ \\
\hline C17: 1 & $0.93 \pm 0.09 b c$ & $1.15 \pm 0.12 b$ & $0.78 \pm 0.03 \mathrm{c}$ & $1.54 \pm 0.16 \mathrm{a}$ & $1.27 \pm 0.25$ & $1.18 \pm 0.31$ & $1.02 \pm 0.26$ & $1.70 \pm 0.38$ \\
\hline C18: $\ln 9 \mathrm{t}$ & $2.01 \pm 0.39 \mathrm{ab}$ & $1.08 \pm 0.34 \mathrm{~b}$ & $2.61 \pm 0.06 \mathrm{a}$ & $1.40 \pm 0.21 \mathrm{~b}$ & $3.65 \pm 0.94$ & $2.00 \pm 0.63$ & $1.54 \pm 0.59$ & $1.53 \pm 0.52$ \\
\hline $\mathrm{C} 18: \ln 9 \mathrm{c}$ & $29.25 \pm 0.79 \mathrm{c}$ & $34.15 \pm 1.17 \mathrm{~b}$ & $28.90 \pm 1.17 \mathrm{c}$ & $40.40 \pm 1.78 \mathrm{a}$ & $27.65 \pm 1.52 \mathrm{c}$ & $33.79 \pm 1.82 \mathrm{~b}$ & $29.33 \pm 1.27 b c$ & $41.18 \pm 1.63 \mathrm{a}$ \\
\hline $\mathrm{C} 18 \mathrm{2n} 6 \mathrm{t}$ & $0.82 \pm 0.05 \mathrm{ab}$ & $0.95 \pm 0.13 \mathrm{a}$ & $0.61 \pm 0.02 \mathrm{~b}$ & $0.93 \pm 0.06 \mathrm{a}$ & $1.38 \pm 0.21$ & $1.13 \pm 0.27$ & $1.37 \pm 0.37$ & $1.15 \pm 0.18$ \\
\hline $\mathrm{C} 18: 2 \mathrm{n} 6 \mathrm{c}$ & $2.43 \pm 0.17 \mathrm{a}$ & $2.00 \pm 0.10 \mathrm{~b}$ & $1.89 \pm 0.08 \mathrm{~b}$ & $1.98 \pm 0.07 \mathrm{~b}$ & $3.35 \pm 0.64$ & $3.08 \pm 0.60$ & $2.63 \pm 0.57$ & $2.98 \pm 0.38$ \\
\hline C18:3n6 & $0.18 \pm 0.04$ & $0.24 \pm 0.09$ & $0.10 \pm 0.02$ & $0.11 \pm 0.02$ & $0.31 \pm 0.19$ & $0.22 \pm 0.12$ & $0.32 \pm 0.12$ & $0.27 \pm 0.14$ \\
\hline $\mathrm{C} 20: 1$ & $0.37 \pm 0.04$ & $0.35 \pm 0.06$ & $0.35 \pm 0.06$ & $0.32 \pm 0.03$ & $0.74 \pm 0.24$ & $0.45 \pm 0.26$ & $0.43 \pm 0.11$ & $0.36 \pm 0.10$ \\
\hline $\mathrm{C} 18: 3 \mathrm{n} 3$ & $0.51 \pm 0.02 \mathrm{a}$ & $0.59 \pm 0.05 \mathrm{ab}$ & $0.43 \pm 0.02 \mathrm{~b}$ & $0.49 \pm 0.03 b$ & $0.75 \pm 0.19$ & $0.45 \pm 0.26$ & $0.48 \pm 0.06$ & $0.35 \pm 0.10$ \\
\hline $\mathrm{C} 20: 2$ & $0.31 \pm 0.11$ & $0.31 \pm 0.02$ & $0.35 \pm 0.28$ & $0.14 \pm 0.04$ & $0.92 \pm 0.50$ & $0.66 \pm 0.34$ & $0.31 \pm 0.11$ & $0.19 \pm 0.07$ \\
\hline $\mathrm{C} 20: 3 \mathrm{n} 6$ & $0.09 \pm 0.04$ & $0.11 \pm 0.06$ & $0.15 \pm 0.13$ & $0.17 \pm 0.13$ & $0.48 \pm 0.27$ & $0.17 \pm 0.09$ & $0.11 \pm 0.02$ & $0.03 \pm 0.01$ \\
\hline $\mathrm{C} 22: \ln 9$ & $0.13 \pm 0.02$ & $0.22 \pm 0.10$ & $0.20 \pm 0.07$ & $0.13 \pm 0.06$ & $0.28 \pm 0.11 \mathrm{a}$ & $0.08 \pm 0.04 \mathrm{ab}$ & $0.11 \pm 0.03 \mathrm{ab}$ & $0.02 \pm 0.01 \mathrm{~b}$ \\
\hline $\mathrm{C} 20: 3 \mathrm{n} 3$ & $0.02 \pm 0.01$ & $0.11 \pm 0.05$ & $0.02 \pm 0.01$ & $0.05 \pm 0.02$ & $0.21 \pm 0.13$ & $0.04 \pm 0.03$ & $0.02 \pm 0.02$ & $0.02 \pm 0.00$ \\
\hline $\mathrm{C} 20: 4 \mathrm{n} 6$ & $0.10 \pm 0.01$ & $0.14 \pm 0.04$ & $0.07 \pm 0.04$ & $0.10 \pm 0.03$ & $0.22 \pm 0.10$ & $0.11 \pm 0.02$ & $0.14 \pm 0.06$ & $0.27 \pm 0.17$ \\
\hline $\mathrm{C} 22: 2$ & $0.02 \pm 0.00$ & $0.11 \pm 0.06$ & $0.03 \pm 0.02$ & $0.03 \pm 0.01$ & $0.13 \pm 0.11$ & $0.03 \pm 0.01$ & $0.05 \pm 0.02$ & $0.40 \pm 0.38$ \\
\hline $\mathrm{C} 20: 5 \mathrm{n} 3$ & $0.02 \pm 0.00$ & $0.05 \pm 0.02$ & $0.04 \pm 0.03$ & $0.03 \pm 0.01$ & $0.01 \pm 0.01$ & $0.02 \pm 0.02$ & $0.38 \pm 0.35$ & $0.08 \pm 0.05$ \\
\hline C24:1 & $0.07 \pm 0.03$ & $0.11 \pm 0.05$ & $0.02 \pm 0.01$ & $0.03 \pm 0.02$ & $0.17 \pm 0.14$ & $0.02 \pm 0.00$ & $0.22 \pm 0.19$ & $0.24 \pm 0.13$ \\
\hline $\mathrm{C} 22: 6 \mathrm{n} 3$ & $0.16 \pm 0.03$ & $0.24 \pm 0.09$ & $0.16 \pm 0.06$ & $0.45 \pm 0.23$ & $0.25 \pm 0.08$ & $0.29 \pm 0.23$ & $0.35 \pm 0.24$ & $0.21 \pm 0.06$ \\
\hline SFA & $58.15 \pm 0.89 a$ & $53.37 \pm 0.94 \mathrm{~b}$ & $59.34 \pm 0.88 \mathrm{a}$ & $46.77 \pm 1.99 \mathrm{c}$ & $53.38 \pm 2.69 \mathrm{a}$ & $51.81 \pm 1.62 \mathrm{a}$ & $57.05 \pm 2.81 \mathrm{a}$ & $43.31 \pm 0.33 b$ \\
\hline UFA & $41.84 \pm 0.89 c$ & $46.62 \pm 0.94 \mathrm{~b}$ & $40.66 \pm 0.88 c$ & $53.23 \pm 1.99 a$ & $46.61 \pm 2.69 b$ & $48.20 \pm 1.60 \mathrm{~b}$ & $42.97 \pm 1.13 b$ & $56.68 \pm 0.33 a$ \\
\hline MUFA & $37.17 \pm 0.71 \mathrm{c}$ & $41.78 \pm 0.94 \mathrm{~b}$ & $36.79 \pm 1.03 \mathrm{c}$ & $48.74 \pm 1.84 a$ & $38.59 \pm 1.63 \mathrm{cb}$ & $41.86 \pm 1.20 \mathrm{~b}$ & $36.80 \pm 0.84 \mathrm{c}$ & $50.60 \pm 2.53 \mathrm{a}$ \\
\hline PUFA & $4.66 \pm 0.29$ & $4.84 \pm 0.53$ & $3.87 \pm 0.63$ & $4.49 \pm 0.37$ & $8.01 \pm 1.49$ & $6.34 \pm 0.96$ & $6.18 \pm 0.78$ & $6.08 \pm 1.00$ \\
\hline n-3PUFA & $1.06 \pm 0.08$ & $1.22 \pm 0.17$ & $0.98 \pm 0.13$ & $1.29 \pm 0.24$ & $1.76 \pm 0.45$ & $1.34 \pm 0.40$ & $1.65 \pm 0.38$ & $1.12 \pm 0.22$ \\
\hline n-6PUFA & $3.84 \pm 0.24$ & $3.64 \pm 0.36$ & $3.03 \pm 0.41$ & $3.27 \pm 0.14$ & $6.18 \pm 0.92$ & $5.19 \pm 0.76$ & $4.77 \pm 0.63$ & $4.86 \pm 0.55$ \\
\hline
\end{tabular}

注: 表达量标注相同小字母表示差异不显著 $(P>0.05)$; 标注不同小写字母表示差异显著 $(P<0.05)$, 仅标注了不同品种羊各部位之间的差异性。

\section{表3显示:}

1) SFA在藏绵羊肾周脂肪、大网膜脂肪含量高于皮下 脂肪、尾部脂肪（P<0.05）, 同时在兰州大尾羊肾周脂肪 皮下脂、大网膜脂肪、肾周脂肪含量高于尾部脂肪 $(\mathrm{P}<0.05)$, 尾部脂肪UFA含量高于SFA; SFA组成特点为: 不同绵羊主要以 $\mathrm{C} 18: 0 、 \mathrm{C} 16: 0$ 为主要脂肪酸, $\mathrm{C} 18: 0$ 含量 在兰州大尾羊的肾周脂肪、大网膜脂肪、皮下脂肪、尾部 脂肪占总脂肪酸含量分别是 $28.15 \% 、 24.18 \% 、 19.94 \%$ 、 $11.37 \%$, 在藏绵羊的肾周脂肪、大网膜脂肪、皮下脂肪、 尾部脂肪占总脂肪酸含量分别是 $26.74 \%$ 、 $25.24 \% 、 18.30 \%$ 、 13. $20 \%$, 肾周脂肪和大网膜脂肪 C18:0含量比皮下脂肪和 尾部脂肪高, $\mathrm{C} 16: 0$ 在藏绵羊各脂肪组织中差异不显著
$(P>0.05)$, 在兰州大尾羊中皮下脂肪中含量较高 $(\mathrm{P}<0.05)$ 。

2) UFA在兰州大尾羊、藏绵羊不同脂肪组织含量有 尾部脂肪>皮下脂肪>大网膜脂肪> 肾周脂肪, 且尾部脂肪 比肾周脂肪UFA含量高 $(P<0.05)$, UFA按照含有单个不 饱和键和多个不饱和键分为MUFA和PUFA, 本试验中检 测了 9 种MUFA和 11 种PUFA, 在兰州大尾羊、藏绵羊中, MUFA含量有尾部脂肪 $>$ 皮下脂肪 $>$ 大网膜脂肪 $>$ 肾周脂肪, 且尾部脂肪比肾周脂肪MUFA含量高 $(P<0.05)$; 兰州大尾 羊和藏绵羊的MUFA组成特点为: C18:1含量最高, 同时 尾部脂肪、皮下脂肪有 $\mathrm{C} 18$ : 1 含量高于大网膜脂肪或肾周 脂肪 $(P<0.05)$, PUFA含量在兰州大尾羊、藏绵羊各脂 肪组织中含量相似 $(P>0.05)$ 。 
3) PUFA与SFA含量比值, 简称P:S值, 它反应了 PUFA 的丰富程度, 在兰州大尾羊各脂肪组织中, $\mathrm{P}: \mathrm{S}$ 比值差异 不显著 $(P>0.05)$, 在藏绵羊各脂肪组织之间, 尾部脂肪 和肾周脂肪之间P:S比值差异显著 $(P<0.05)$; 同时, 兰 州大尾羊脂肪组织中的n-6系PUFAs和n-3系PUFAs的脂肪 酸含量高于藏绵羊, 两种脂尾型绵羊都有n-6系PUFAs特 征。

\section{4. 讨论}

\section{1. 不同脂尾型绵羊体脂分布分析}

本试验通过比较, 8 月龄三种绵羊在宰前活重没有明 显差异条件下, 藏绵羊、兰州大尾羊胴体重比小尾寒羊更 高, 然而兰州大尾羊尾脂占胴体比例高, 在尾脂没有得到 有效利用的条件下, 藏绵羊屠宰性能更优, 更具有较高的 经济价值, 同时结果表明脂尾型绵羊大网膜和尾部脂肪含 量更高, 庋脂尾型绵羊肾周、大网膜含量更高。

\section{2. 不同脂尾型绵羊脂肪组织大小和粗脂肪含量分析}

$\mathrm{Ju}$ 等 [7-8]人研究发现单个脂肪细胞的体积与脂肪细 胞分化程度和甘油三酯积累量有关, 本研究中三种脂尾型 绵羊脂肪组织大小比较发现脂尾型绵羊兰州大尾羊、小尾 寒羊皮下脂肪周长比大网膜、肾周脂肪大, 庋脂尾型绵羊 脂肪组织大网膜脂肪比皮下脂肪大且皮下组织与肾周脂 肪差异不显著, 同时各脂肪组织粗脂肪含量差异也不显著, 推测䧹脂尾型藏绵羊属于山地型绵羊, 为保护内脏器官, 在进化过程中内脏脂肪发育早、分化程度高, 细胞体积大, 同时皮下积累大量脂肪, 有利于低于严寒, 这可能是造成 藏绵羊皮下脂肪和肾周脂肪差异不显著的原因, 而兰州大 尾羊等绵羊脂尾型性状是在极端自然环境下获得 [9～10], 尤其冬季饲草资源匮乏的黄土高原地区，皮下储存能量有 利于降低脂肪对内脏器官的脂毒性, 并且利于保温, 这可 能造成脂尾型绵羊皮下脂肪分化程度高的原因, 本研究同 时发现脂尾型绵羊脂肪细胞小于庡脂尾绵羊，韩吉龙[11] 研究发现成年脂尾型绵羊尾部细胞大于䧹脂尾型面积, 庋 脂尾型绵羊脂肪 3 月龄脂肪细胞大于成年，何晓军等[12] 人研究发现兰州大尾羊尾脂在 3 月龄、9月龄有两个生长高 峰期, 刘政等[13]人认为脂肪肥大是细胞增大和数目增多, 推测脂尾型绵羊在成年后由于甘油三酯积累造成尾部脂 肪细胞肥大, 在此月龄还是以细胞数目增加为主, 同时度 脂尾型尾部脂肪细胞大于脂尾型绵羊, 再次证明幼年度尾 型绵羊尾脂成熟早。

\section{3. 不同脂尾型绵羊脂肪酸组成分析}

反刍家畜脂肪酸代谢与单室胃动物不同, 由于瘤胃内 环境处于高度的还原性, 脂类物质水解产生的UFA会被氢 化菌氧化为SFA[14], 经过淋巴管吸收, 到达小肠粘膜细胞 后, 转化为乳糜颗粒和低密度脂蛋白胆固醇, 被机体吸收 和利用 [15-16], 幼年反刍动物由于瘤胃微生物环境未完全 形成, 瘤胃微生物氧化能力较弱, 随着年龄增长, 瘤胃内 氢化菌增多, 瘤胃容积扩大, 将UFA氧化为SFA的能力增
强[17], 本试验发现SFA在藏绵羊肾周脂肪、大网膜脂肪 含量高于皮下脂肪、尾部脂肪, 同时在兰州大尾羊皮下脂、 大网膜脂肪、肾周脂肪含量高于尾部脂肪, 尾部脂肪UFA 含量高于SFA，这可能与脂肪沉积顺序有关，杨东等 [18] 人研究发现羊脂肪先沉积腹部脂肪、皮下脂肪、肌间脂肪、 肌内脂肪, 由于腹部脂肪先沉积、皮下脂肪后沉积, 在羔 羊发育过程中可能会造成内脏脂肪SFA含量高于皮下脂 肪, 同时内脏温度高, 有利于UFA氧化为SFA, 尾部远离 心脏, 体温低, 不利于UFA向SFA转化, 造成UFA在兰州 大尾羊、藏绵羊不同脂肪组织含量有尾部脂肪>皮下脂肪> 大网膜脂肪>肾周脂肪, 本研究也发现两种绵羊各脂肪组 织UFA含量差异不明显, 说明UFA可能与尾型形状无关。 硬脂酸 $\mathrm{C} 18: 0$ 在动物脂肪内含量较高, 本研究两种绵羊硬 脂酸在肾周脂肪和大网膜脂肪含量比皮下脂肪和尾部脂 肪高, 且尾部脂肪中含量最低, 尾部脂肪也属于皮下脂肪, 因此硬脂酸在皮下脂肪组织中表现出明显的差异性, 这一 研究与吴建平[19]研究结果相同, 研究发现硬脂酸易发生 不饱和作用, 这可能由于 $118: 0$ 发生了不饱和化作用, 造 成C18:0含量降低,

研究表明UFA脂肪酸可以使胆固醇发生酯化反应, 降 低胆固醇水平, 减少动脉血管发生弹样硬化发生率, 提高 血管通畅性, 改善血流循环, 同时具有维持细胞膜结构稳 定性作用, 减少神经细胞死亡, 提高记忆力的作用 [15]。 油酸作为主要的一种UFA, 其能降低有害胆固醇, 对有益 胆固醇没有作用, 较好的改变肉质风味[20]。在兰州大尾 羊、藏绵羊中, MUFA含量有尾部脂肪>皮下脂肪>大网膜 脂肪>肾周脂肪, 且尾部脂肪和肾周脂肪MUFA含量相比 差异性显著, 这是由于兰州大尾羊和藏绵羊的 MUFAC18:1含量最高, 同时有尾部脂肪、皮下脂肪的 $\mathrm{C} 18: 1$ 含量高于大网膜脂肪或肾周脂肪, $\mathrm{C} 18: 1$ 可以较好的 改变肉的风味, 报到指出MUFA的功能主要体现在 $\mathrm{C} 18: 1$ 中, $\mathrm{C} 18: 1$ 皮下脂肪中含量较多, 有利于改善肉质品质, C18:1含量在两种绵羊中有相似的体脂分布特征, 同时 PUFA含量在兰州大尾羊、藏绵羊各脂肪组织中含量差异 不显著, 说明两种不同尾型的绵羊在主要UFA的代谢上可 能存在共同的特征。

人类饮食健康中, $\mathrm{P}: \mathrm{S}$ 比值最好高于 $0.4[21]$, 家畜肉 产品自然P:S比值大约在0.1左右 [15], 本研究所测各脂肪 组织的P:S比例于此相吻合, 提高P:S的值可以提高肉质品 质, 在兰州大尾羊各脂肪组织中, $\mathrm{P}: \mathrm{S}$ 比值差异不显著, 但尾脂P:S比值为 0.14 , 较其他部位PUFA含量更高, 虽然 在藏绵羊各脂肪组织之间, 尾部脂肪和肾周脂肪间P:S比 值差异显著, 但最高P:S值为 0.1 , 说明兰州大尾羊尾脂更 具有提高PUFA的潜力。膳食中 $\omega$-3PUFAs的含量对代谢综 合症、心血管疾病、免疫学功能和肿瘤等疾病的发生具有 重要作用[21-22],n-6PUFA与脂代谢、免疫反应有关, 人体 健康需要n-6系PUFAs和n-3系PUFAs的平衡来维持[23], 兰 州大尾羊脂肪组织中的n-6系PUFAs和n-3系PUFAs的脂肪 酸含量均高于藏绵羊, 两种尾型绵羊都有n-6系PUFAs营 养特征, n-6系PUFAs和n-3系PUFAs代表了肉中必须脂肪 酸含量, 同样说明不同脂尾型绵羊在必须脂肪酸代谢上存 在相同特点, 研究也表明n-6系PUFAs和n-3系PUFAs含量 
与精饲料饲喂有关[24], 本试验在动物饲喂过程中均添加 了精料, 但由于不同羊种的吸收能力、生长环境变化等是 否会影响各脂肪组织脂肪酸含量, 需进一步研究。

\section{5. 结论}

8月龄不同脂尾型绵羊比较发现: 尾型绵羊以尾部、 皮下、大网膜富集脂肪, 而度脂尾型绵羊以内脏、皮下富 集脂肪; 脂尾型绵羊皮下脂肪发育早, 分化程度高, 尾脂 发育周期长, 庋尾型皮下和肾周脂肪有相似分化发育的特 征, 尾脂成熟早; 兰州大尾羊和藏绵羊主要脂肪酸具有相 似的部位分布特征。

\section{致谢}

本文为国家自然基金一般项目《不同脂尾型绵羊脂肪 性状相关基因篎选及分子调控机理研究》(31360529)的阶 段性成果之一。

\section{参考文献}

[1] Davoli R, Braglia S. Molecular approaches in pig breeding to improve meat quality[J]. Briefings in Functional Genomics \& Proteomics, 2007, 6(4):313.

[2] 韦璇.LPL、 $\mathrm{C} / \mathrm{EBP} \alpha$ 基因在不同尾型绵羊尾部脂肪组织中的 表达分析[D].西北农林科技大学,2014。

[3] 徐红伟,䟵荣金杨,具田,等.兰州大尾羊遗传资源保护与开发 利用[J].中国畜牧兽医,2009,36(8):88-90。

[4] Marai I F M, Daader A H, Bahgat L B. Performance traits of purebred Ossimi and Rahmani lambs and their crosses with Finnsheep born under two accelerated mating systems.[J]. Archiv Tierzucht, 2009, 52(5):497-511.

[5] 张伟,沈敏,李欢,等.绵羊X染色体59571364与59912586位点 在脂尾、瘦尾绵羊群体中的多态性检测及分析 [J]. 遗 传,2013,35(12):1384-1390。

[6] 张明.安格斯与西门塔尔牛杂交一代育肥性能及肉品质研 究[D].甘肃农业大学, 2016。

[7] Ju D P, Zhan L X. Developments in Regulation of Adipocytes Differentiation[J]. Chinese Journal of Cell Biology, 2010, 32(5):690-695.

[8] Rosen E D, Macdougald O A. Adipocyte differentiation from the inside out[J]. Nature Reviews Molecular Cell Biology, 2006, 7(12):885-96.
[9] Moradi M H, Nejati-Javaremi A, Moradi-Shahrbabak M, et al. Genomic scan of selective sweeps in thin and fat tail sheep breeds for identifying of candidate regions associated with fat deposition[J]. BMC Genetics, 2012, 13(1):1-15

[10] Marai I F M, Daader A H, Bahgat L B. Performance traits of purebred Ossimi and Rahmani lambs and their crosses with Finnsheep born under two accelerated mating systems.[J]. Archiv Tierzucht, 2009, 52(5):497-511.

[11] 韩吉龙.脂尾型绵羊尾部脂肪富集的蛋白质组学研究[D]. 中国农业科学院, 2016。

[12] 何晓军,㶓荣金金，许小红,等. 兰州大尾羊尾部脂肪细胞发育 性变化研究 $[J]$. 中国草食动物科学, 2012, 32(4):8-10。

[13] 刘政, 赵生国, 李华伟,等. 脂尾去除对“兰州大尾羊”和“蒙 古羊'生长性能及脂肪沉积分布的影响 [J]. 中国农学通报, 2015, 31(5):7-11。

[14] Chikwanha O C, Vahmani P, Muchenje V, et al. Nutritional enhancement of sheep meat fatty acid profile for human health and wellbeing $[\mathrm{J}]$. Food Research International, 2017.

[15] 刘婷. 牦牛黄牛体脂脂肪酸营养特征及温度对其影响的研 究[D]. 甘肃农业大学, 2010。

[16] Liu T, Lei Z M, Wu J P, et al. Fatty acid composition differences between adipose depot sites in dairy and beef steer breeds[J]. Journal of Food Science \& Technology, 2015, 52(3):1656-1662.

[17] 张利平, 吴建平. 肉羊体脂脂肪酸与肉品质关系的研究 [J]. 甘肃农大学报, 2000, 35(4):363-369。

[18] 杨东,王文义,乔文,等.肉羊脂肪沉积及其调控手段 [J].粮食 与饲料工业,2016,12(1):51-55。

[19] 吴建平.不同肉羊品种体脂脂肪酸遗传变异性及其特性的 研究[D].甘肃农业大学, 2000。

[20] 曹芝.内蒙古不同杂交品种肉牛生产性状比较研究[D]. 内 蒙古农业大学, 2012。

[21] 王珊珊, 李秋,徐田涁, 等. $\omega$-3多不饱和脂肪酸的生理功能特 性及应用[J].中国食物与营养, 2009, 2009(10):51-54。

[22] Gao Y, Qu Y, Luo H. Research Progress for Effect of n-3 Polyunsaturated Fatty Acids on Semen Quality[J]. Modern Journal of Animal Husbandry \& Veterinary Medicine, 2017.

[23] 双金,敖力格日玛,敖长金.苏尼特羊体脂脂肪酸组成的研究 [J]. 畜牧兽医学报, 2015, 46(8):1363-1374。

[24] 赵天章.日粮油脂类型对羊肉脂肪酸和肌内脂肪含量的影 响及其机理[D]. 中国农业大学, 2014。 